\title{
Single Nucleotide Polymorphisms in Chemosensory Pathway Genes GNB3, TAS2R19, and TAS2R38 Are Associated with Chronic Rhinosinusitis
}

\author{
Phillip R. Purnell ${ }^{\mathrm{a}}$ Benjamin L. Addicks ${ }^{\mathrm{a}}$ Habib G. Zalzal $^{\mathrm{a}}$ Scott Shapiro $^{\mathrm{a}}$ \\ Sijin Wen ${ }^{b}$ Hassan H. Ramadan ${ }^{a}$ Vincent Setolac ${ }^{c-e}$ David P. Siderovski ${ }^{c}$ \\ ${ }^{a}$ Department of Otolaryngology, Head and Neck Surgery, West Virginia University School of Medicine, Morgantown, \\ WV, USA; ' Department of Biostatistics, West Virginia University School of Public Health, Morgantown, WV, USA; \\ 'Department of Physiology and Pharmacology, West Virginia University School of Medicine, Morgantown, WV, USA; \\ ${ }^{d}$ Department of Neuroscience, West Virginia University School of Medicine, Morgantown, WV, USA; ${ }^{\text {Department of }}$ \\ Behavioral Medicine and Psychiatry, West Virginia University School of Medicine, Morgantown, WV, USA
}

\section{Keywords}

Bitterant signaling · Chronic rhinosinusitis - Genetics · Innate immune response $\cdot$ Single nucleotide polymorphism

\begin{abstract}
Background: Chronic rhinosinusitis (CRS) is a multifaceted disease with a significant genetic component. The importance of taste receptor signaling has recently been highlighted in CRS; single nucleotide polymorphisms (SNPs) of bitter tastant-responsive G-protein-coupled receptors have been linked with CRS and with altered innate immune responses to multiple bacterially derived signals. Objective: To determine in CRS the frequency of six SNPs in genes with known bitter tastant signaling function. Methods: Genomic DNA was isolated from 74 CRS volunteers in West Virginia, and allele frequency was determined and compared with demographically matched data from the 1,000 Genomes database. Results: For two SNPs in a gene recently associated with bitterant signaling regulation, RGS21, there were no associations with CRS (although the frequency of the minor allele of RGS21, rs7528947, was seen to increase with increasing Lund-Mackay CT staging score). Two TAS2R bitter
\end{abstract}

() 2019 S. Karger AG, Basel taste receptor gene variants (TAS2R19 rs10772420 and TAS2R38 rs713598), identified in prior CRS genetics studies, were found to have similar associations in this study. Conclusion: Unique to our study is the establishment of an association between CRS in this patient population and GNB3 SNP rs5443, a variation in an established $\mathrm{G}$ protein component downstream of bitterant receptor signal transduction.

๑) 2019 S. Karger AG, Basel

\section{Introduction}

Chronic rhinosinusitis (CRS) is a persistent inflammatory state of the sinus mucosa with symptoms of nasal obstruction or discharge with or without facial pain and changes in smell present for more than 12 weeks [1]. Prevalence estimates range from 10 to $12 \%$ of the population in the USA and Europe [2], resulting in health care costs greater than USD 770 million in the USA alone [3]. CRS patients have poor quality of life scores, similar to

Edited by: I. Lehmann, Berlin.

\section{KARGER}

E-Mail karger@karger.com

www.karger.com/iaa
Prof. David P. Siderovski

Department of Physiology and Pharmacology

West Virginia University School of Medicine

64 Medical Center Drive, Morgantown, WV 26506-9229 (USA)

E-Mail dpsiderovski@hsc.wvu.edu 
other chronic diseases, including CHF and COPD [4]. Evidence suggests a genetic component to CRS [5]. In a large population study, there was a 2.4 -fold increased risk of CRS in first-degree relatives of patients [6]; this number is higher for first-degree relatives of patients with nasal polyps. Furthermore, several well-characterized genetic syndromes are associated with CRS, including cystic fibrosis [7] and primary ciliary dyskinesia [8].

An emerging concept regarding the chronic nature of CRS relates to a potential deficit in sensing and clearing bacterial infections; receptors for bitter and sweet taste have been found in the airway and are thought to play a role in sensing bacteria and regulating innate immune responses [9]. Bitter taste is initiated in the oral cavity through members of the T2R family of $\mathrm{G}$ protein-coupled receptors (GPCRs) [10-12]. However, T2R bitter receptors are not limited to taste bud cells; they are also found in solitary chemosensory cells and ciliated epithelial cells of the nasal and sinus cavities, functioning in these locales to "taste" secreted bacterial products, such as acyl-homoserine lactones [13]. Gram-negative bacteria, such as Pseudomonas aeruginosa, use acyl-homoserine lactones as quorum-sensing molecules [14]. Binding of these bacterial products to T2Rs is thought to modulate innate host immune responses, such as release of antimicrobial peptides and nitric oxide.

Genome-wide association studies (GWAS) have identified several genes potentially associated with CRS $[15,16]$. Recent genetic studies have underscored the importance of taste receptor signaling in innate immunity of the upper and lower airways, and paranasal sinuses [13]; single nucleotide polymorphisms (SNPs) in taste receptor genes have since been associated with altered bacterial immune response and, thus, with CRS $[17,18]$. There are 25 type 2 taste receptors (TAS2R "bitterant receptors"), coupled to $\mathrm{G}$ protein signaling, that are expressed in multiple tissues. In the ciliated mucosa of the sinuses, these TAS2Rs respond to chemoirritants and bacterially produced secretions [19]. Some of us also recently showed that an inhibitor of GPCR signaling, regulator of G-protein signaling-21 (RGS21), opposes TAS2R-mediated bitterant signaling in immortalized airway epithelial cells and, given its expression in sinus mucosa and airway epithelia, may be involved in mucociliary clearance $[20,21]$. Here, investigating candidate genes involved in bitterant signaling from among the TAS2R genes and RGS21, we identified three SNPs more common in West Virginia CRS patients than in healthy controls of European descent, including an SNP within GNB3, a downstream component of bitterant signaling previously unassociated with CRS.

Variations in Chemosensory Pathway

Genes Associated with CRS
Table 1. Patient demographics (total $n=74$ patients)

\begin{tabular}{lr}
\hline Mean age, years & 55.0 \\
Male & $38(52)$ \\
Female & $36(48)$ \\
Allergy & $42(57)$ \\
Asthma & $28(38)$ \\
Migraine & $8(11)$ \\
Current smoker & $9(11)$ \\
History of smoking & $19(26)$ \\
Polyps & $45(61)$ \\
Previous surgery & $29(39)$ \\
\hline
\end{tabular}

Lund-Mackay score $(\mathrm{n}=66$ patients)

$\begin{array}{ll}1-8 & 22(33) \\ 9-16 & 29(44) \\ 17-24 & 15(23) \\ \text { Average score } & 11.6[10.0-13.3]\end{array}$

SNOT22

Total $(0-110) \quad 49.0[43.5-54.6]$

Rhinologic symptoms (0-30) 13.0 [11.4-14.5]

Extranasal rhinologic

symptoms $(0-15) \quad 7.0[6.1-8.0]$

Ear/facial symptoms (0-25) 9.7 [8.4-11.1]

Psychological dysfunction (0-35) 17.5 [15.1-19.8]

Sleep dysfunction (0-25)

$12.4[10.8-14.0]$

Data are presented as $n$ (\%) or average score [CI: 5-95\%]. In SNOT22, the score range is indicated in parentheses.

\section{Materials and Methods}

\section{Recruitment}

Seventy-four volunteers were seen at an academic tertiary referral center in West Virginia. The study was approved by the Institutional Review Board of West Virginia University (WVU) Office of Research Integrity and Compliance. All volunteers met EPOS criteria [22] for CRS, and informed consent was obtained under IRB 1410476782. Age, sex, allergy, asthma, migraine, smoking status, presence of polyps, and previous surgical history were recorded (Table 1). CT scans of the sinuses were available for 66 of the 74 patients. Lund-Mackay CT score was calculated based on the standard radiologic staging of CRS for these patients [23]. Sino-nasal Outcome Test-22 (SNOT22) scores were obtained from all patients; total SNOT22 and the five standard quality of life domain scores were calculated (Table 1) [24]. Patients with cystic fibrosis, primary ciliary dyskinesia, known immunodeficiency, and craniofacial abnormalities were excluded from the study population.

\section{Tissue Collection and DNA Extraction}

Volunteers provided two buccal swabs, which were each placed in a $15-\mathrm{mL}$ polystyrene conical centrifuge tube and stored at $-80^{\circ} \mathrm{C}$ until DNA extraction using QIAamp DNA Mini Kits (exactly as per the manufacturer's protocol). Purity and yield were assessed by $260 / 280 \mathrm{~nm}$ absorbance on a QIAxpert microfluidic spectrophotometer. 
Table 2. Gene variant minor allele frequency (MAF) data obtained from West Virginia CRS clinic patients $(n=$ 74) and public databases with demographically matched cohorts

\begin{tabular}{llllll}
\hline Gene & dbSNP ID & $\begin{array}{l}\text { Minor } \\
\text { allele }\end{array}$ & $\begin{array}{l}\text { TaqMan } \\
\text { probe }\end{array}$ & European MAF* & MAF in CRS probands \\
\hline TAS2R38 & rs713598 & C & C_8876467_10 & 0.423 & $0.568 p<0.001$ \\
GNB3 & rs5443 & T & C_2184734_10 & $0.367\left(\right.$ HapMap $\left.^{\#}\right)$ & $0.493 p=0.014$ \\
TAS2R19 & rs10772420 & A & C_1317426_10 & 0.505 & $0.601 p=0.024$ \\
TAS2R20 & rs12226920 & T & C_1326611_10 & 0.383 & $0.458 p=0.062$ \\
RGS21 & rs7528947 & G & C_30007846_20 & 0.516 & $0.547 p=0.461$ \\
RGS21 & rs1175152 & A & C_68684_10 & 0.433 & $0.439 p=0.875$ \\
\hline
\end{tabular}

* Minor allele frequency reported on $n=1,006$ Europeans via 1,000 Genomes ( ${ }^{*}$ except as otherwise noted: i.e., 116 Europeans from HapMap); MAF comparisons between West Virginia probands and European SNP databases were previously established in Kaski et al. [44] (2019). ${ }^{\ddagger} p$ value denotes significance of difference between MAFs derived by comparison between CRS probands and European MAF using binomial test (null hypothesis is "no difference between CRS probands and Europeans"; alternative is "there is difference between CRS probands and Europeans."

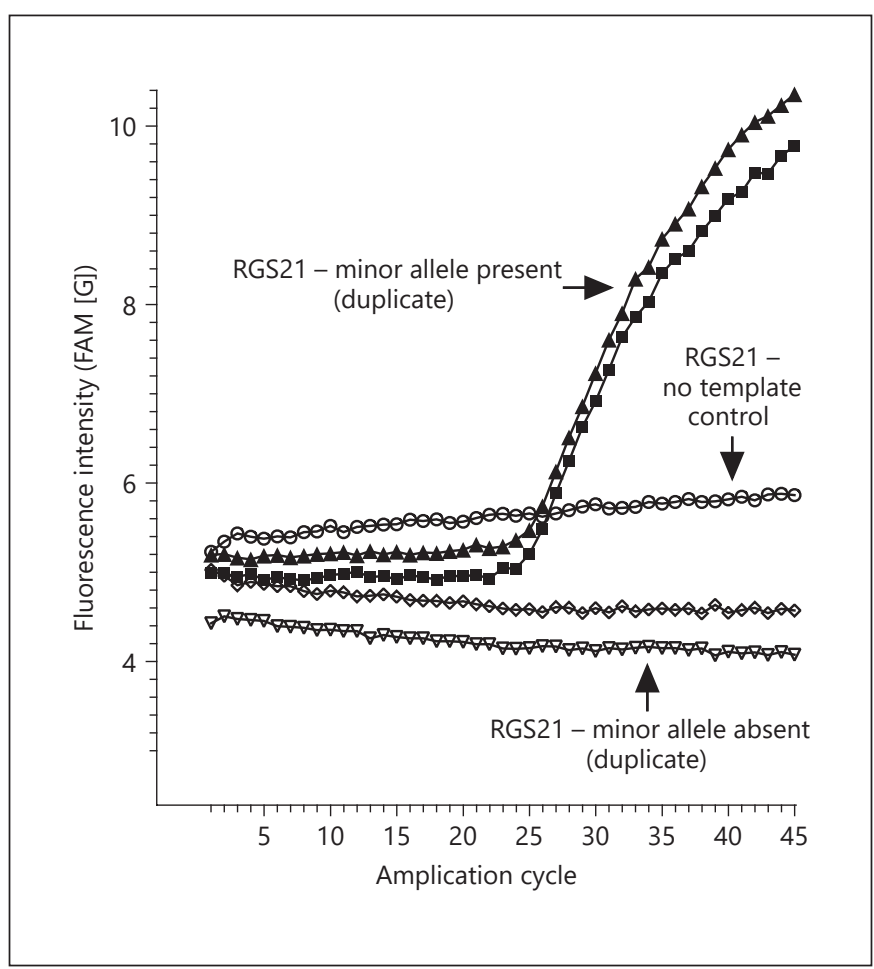

Fig. 1. Sample real-time quantitative polymerase chain reaction (qPCR) results for the detection of the absence or presence of RGS21 SNP rs7528947 (minor allele G) within West Virginia CRS patient genomic DNA using TaqMan primer-probe set C_30007846_20. Examples of probands with the minor allele (closed symbols) and lacking the minor allele (open triangles and open diamonds) are shown; a separate $\mathrm{qPCR}$ reaction lacking input genomic DNA is also illustrated (open circles).

\section{Genotyping}

Genotyping was performed using TaqMan primer-probe sets (Table 2) and Type-it Fast SNP Probe PCR Kits (Qiagen) exactly as per the manufacturer's protocols. Reactions were run on a Qiagen Rotor-Gene Q in duplicate; no-DNA negative control reactions were also performed for each primer-probe set (representative data obtained are displayed in Fig. 1).

\section{Statistical Analyses}

Allele frequencies were compared with 1,006 Europeans (1,000 Genomes Project) [25], given that West Virginia is demographically homogenous [26], i.e., 94\% White European ancestry (http:// censusviewer.com/state/WV). Given no information for the frequency of the GNB3 SNP rs5443 in 1,000 Genomes, its allele frequency was instead compared with 116 Europeans (CEU) from HapMap [27]. Statistical analyses of SNP frequencies were performed using Pearson's $\chi^{2}$. A $p$ value $<0.05$ was used to infer that the allele frequency from CRS patients is significantly different from demographically matched, public genome data. Subgroup analyses were performed comparing the SNP minor allele frequency (MAF) in patients with (CRSwNP) or without (CRSsNP) nasal polyps, and separately based on Lund-Mackay CT score, using standard $\chi^{2}$ statistical tests (Tables 3,4 ).

\section{Results}

Given our recent findings that a negative regulator of bitterant GPCR signaling, RGS21, is expressed in sinus mucosa and airway epithelia $[20,21]$, we interrogated the status within a CRS patient population of two SNPs within RGS21, as well as four SNPs within other bitterant signaling genes with established or unknown association with CRS (i.e., TAS2R19, TAS2R20, TAS2R38, and GNB3). 
Table 3. Gene variant minor allele frequency (MAF) data from CRS patients with and without nasal polyps $(n=74)$.

\begin{tabular}{llllll}
\hline Gene & dbSNP ID & $\begin{array}{l}\text { Minor } \\
\text { allele }\end{array}$ & $\begin{array}{l}\text { CRSwNP } \\
\text { MAF }\end{array}$ & $\begin{array}{l}\text { CRSsNP } \\
\text { MAF }\end{array}$ & $p$ value \\
\hline TAS2R38 & rs713598 & C & 0.567 & 0.569 & 0.978 \\
GNB3 & rs5443 & T & 0.522 & 0.448 & 0.380 \\
TAS2R19 & rs10772420 & A & 0.589 & 0.621 & 0.700 \\
TAS2R20 & rs12226920 & T & 0.456 & 0.466 & 0.906 \\
RGS21 & rs7528947 & G & 0.567 & 0.517 & 0.555 \\
RGS21 & rs1175152 & A & 0.389 & 0.517 & 0.125 \\
\hline
\end{tabular}

Table 4. Gene variant minor allele frequency (MAF) data from CRS based on Lund-Mackay (LM) CT score $(n=66)$

\begin{tabular}{lllllll}
\hline Gene & dbSNP ID & \multicolumn{2}{l}{ Minor LM } \\
allele & $\begin{array}{l}\text { LM } \\
\text { MAF }\end{array}$ & $\begin{array}{l}\text { 9-16 } \\
\text { MAF }\end{array}$ & $\begin{array}{l}\text { 17-24 } \\
\text { MAF }\end{array}$ & $\begin{array}{l}p \\
\text { value }\end{array}$ \\
\hline TAS2R38 & rs713598 & C & 0.614 & 0.500 & 0.600 & 0.459 \\
GNB3 & rs5443 & T & 0.568 & 0.517 & 0.467 & 0.688 \\
TAS2R19 & rs10772420 & A & 0.591 & 0.603 & 0.600 & 0.992 \\
TAS2R20 & rs12226920 & T & 0.523 & 0.362 & 0.533 & 0.167 \\
RGS21 & rs7528947 & G & 0.386 & 0.603 & 0.700 & 0.017 \\
RGS21 & rs1175152 & A & 0.500 & 0.431 & 0.333 & 0.364 \\
\hline
\end{tabular}

All chosen SNPs had an MAF greater than $30 \%$ so they would be identifiable in this patient population. Buccal swabs from CRS patients (Table 1) were used for genomic DNA isolation and genotyping.

\section{TAS2R19 rs10772420 (Type 2 Taste Receptor 19)}

TAS2R19 (previously known as TAS2R48) is a GPCR with bitterant taste receptor activity located on chromosome 12. SNP rs 10772420 within TAS2R19 was previously identified as being associated with RSV infections in a GWAS [28] and also identified in a GWAS of Canadian patients with sinusitis [15]. The minor allele (A) causes a missense mutation (arginine 299 to cysteine) in the encoded protein and is associated with intense quinine perception [29]. The "minor" allele is actually more prominent in Europeans (MAF 0.505; Table 2). In the CRS patients in this study, this allele is even more common (MAF $0.601 ; p=0.024) ; 23 \%$ of the CRS patients were homozygous $(\mathrm{A} / \mathrm{A})$ for the minor allele.

\section{TAS2R38 rs713598 (Type 2 Taste Receptor 38)}

TAS2R38, a bitterant receptor key to phenylthiocarbamide perception [30], is expressed in sinonasal cili- ated epithelium [31] and upper airways [32]. TAS2R38 is implicated in innate immunity and the response to Pseudomonas [31]. rs713598 is a common missense SNP in TAS2R38 [30], associated with quinine intensity [29] and other taste preferences [33-35]. rs713598 is one of the most common SNPs in the TAS2R38 gene, causing a missense mutation to the encoded GPCR (alanine 49 to proline) [30]. In the CRS patients in this study, this allele is more common (MAF 0.568; $p<$ 0.001) than in Europeans of the 1,000 Genomes Project (MAF 0.423).

\section{RGS21 rs7528947 (Regulator of G-Protein}

Signaling-21)

RGS21 is a Ga GTPase-accelerating protein expressed in the lingual epithelium, lung, and gastrointestinal tissues [21]; ablation of Rgs 21 in mice blunts bitterant signaling [36]. rs7528947 is a $3^{\prime}$ untranslated region variant of $R G S 21$ with no known disease associations. The rs7528947 MAF in CRS patients is 0.547 , similar to the European MAF $(0.516 ; p=0.461)$. No difference in rs7528947 MAF was observed when stratified between CRS patients with or without nasal polyps (Table 3); however, a significant difference $(p=0.017)$ was observed across the spectrum of Lund-Mackay CT staging scores (Table 4), with the minor allele appearing more frequently with higher Lund-Mackay scores.

\section{RGS21 Intergenic Region rs1175152 (Regulator of \\ G-Protein Signaling-21)}

A second $R G S 21$-associated SNP, rs1175152, is located in the intergenic region between RGS18 and RGS21 and is also of unknown consequence. To date, there have been no known studies identifying this marker with any known phenotype or in association with any disease. Its MAF among Europeans of the 1,000 Genomes Project is 0.433 , similar to the frequency observed in the CRS patients of this study (MAF 0.439; $p=0.875$ ).

\section{TAS2R20 rs12226920 (Type 2 Taste Receptor 20)}

TAS2R20 (also known as TAS2R49) is a bitterant receptor expressed in multiple tissue types [37]. Its missense SNP rs12226920 (histidine 143 to glutamine) was previously associated with CRS, with a biallelic difference of $16 \%$ versus controls, suggesting a possible role for variations in this GPCR in CRS pathogenesis [15]. In the CRS patients of this study, the MAF (0.458) was higher than that predicted by 1,000 Genomes (European MAF 0.383); however, this difference did not meet our statistical significance threshold $(p=0.062)$. 
GNB3 rs5443 (G Protein Subunit Beta 3)

GNB3 encodes a $G \beta$ subunit of the G protein heterotrimer. Its SNP, rs5443, has been studied extensively in other contexts and is associated with numerous metabolic-related diseases, including obesity [38], coronary artery disease, and hypertension [39], as well as the increased efficacy of antidepressants in major depressive disorder [40]. All of these associations are with minor allele $(\mathrm{T})$ carriers. The HapMap European MAF is 0.367, whereas the CRS patients of this study showed an increased MAF of $0.493(p=0.014)$.

\section{Discussion}

While RGS21 is functionally linked to bitterant GPCR signaling [20,21,36], neither RGS21 SNP tested was found in this study to be associated with CRS (although the minor allele of RGS21 SNP rs7528947 was seen more frequently in CRS patients with higher Lund-Mackay CT staging scores). Conversely, GNB3 SNP rs5443, a gene variation in an established component of bitterant GPCR signaling [41] but without a previous association with CRS, was found to be more highly prevalent in West Virginia CRS patients, along with two TAS2R variants previously identified as being associated with CRS. $G \beta$ subunits of $G$ protein heterotrimers, such as that encoded by GNB3, are known to assemble with E3 ubiquitin ligase complexes responsible for degradation of GRK2, a GPCR kinase [42]. Recent work has shown that GNB3 SNP rs5443, identified more commonly in the CRS patients of this study, is associated with decreased GRK2 ubiquitination [43]. Further studies are therefore required to clarify the role of altered GNB3 function (and potentially GRK2 function) in bitterant signaling, innate immunity activation/regulation, and CRS.

\section{Acknowledgments}

We thank the American Academy of Otolaryngology - Head and Neck Surgery Foundation (AAO-HNSF) Annual Meeting in Chicago, IL, USA, September 10-13, 2017, for accepting an oral presentation by one of the authors (B.L.A.) of an interim report on this study.

\section{Statement of Ethics}

The study protocol was approved by the Institutional Review Board of WVU Office of Research Integrity and Compliance. The subjects of this study gave their written informed consent as obtained under WVU IRB 1410476782.

\section{Disclosure Statement}

The authors have no conflicts of interest to declare.

\section{Funding Sources}

This research was supported in part by the National Institute of General Medical Sciences of the National Institutes of Health under Award Number 5U54GM104942-03 to the West Virginia Clinical and Translational Science Institute (to B.L.A. and D.P.S.). Funding support was also provided by the WVU E.J. Van Liere Endowed Medicine Professorship (to D.P.S.). Neither of these sources participated in the preparation of data or the paper.

\section{Author Contributions}

David P. Siderovski: conception and design, data analysis and interpretation, revising the manuscript, final approval, and agreement to be accountable. Phillip R. Purnell: conception and design, acquisition of data, data analysis and interpretation, drafting and revising the article, final approval, and agreement to be accountable. Benjamin L. Addicks: conception and design, acquisition of data, data analysis and interpretation, revising the manuscript, final approval, and agreement to be accountable. Habib G. Zalzal: conception and design, acquisition of data, revising the manuscript, final approval, and agreement to be accountable. Scott Shapiro: conception and design, acquisition of data, revising the manuscript, final approval, and agreement to be accountable. Sijin Wen: data analysis and interpretation, revising the manuscript, final approval, and agreement to be accountable. Hassan H. Ramadan: conception and design, revising the manuscript, final approval, and agreement to be accountable. Vincent Setola: conception and design, data interpretation, revising the manuscript, final approval, and agreement to be accountable.

\section{References} Alobid I, Baroody F, et al. EPOS 2012: european position paper on rhinosinusitis and nasal polyps 2012. A summary for otorhinolaryngologists. Rhinology. 2012 Mar;50(1):1-12.

2 DeConde AS, Soler ZM. Chronic rhinosinusitis: epidemiology and burden of disease. Am J Rhinol Allergy. 2016 Mar-Apr;30(2):134-9.

3 Bhattacharyya N. Incremental health care utilization and expenditures for chronic rhinosinusitis in the United States. Ann Otol Rhinol Laryngol. 2011 Jul;120(7):423-7.

4 Gliklich RE, Metson R. The health impact of chronic sinusitis in patients seeking otolaryngologic care. Otolaryngol Head Neck Surg. 1995 Jul;113(1):104-9.

5 Yoo F, Suh JD. What is the evidence for genetics in chronic rhinosinusitis? Curr Opin Otolaryngol Head Neck Surg. 2017 Feb;25(1):5463.

Purnell/Addicks/Zalzal/Shapiro/Wen/ Ramadan/Setola/Siderovski 
6 Oakley GM, Curtin K, Orb Q, Schaefer C, Orlandi RR, Alt JA. Familial risk of chronic rhinosinusitis with and without nasal polyposis: genetics or environment. Int Forum Allergy Rhinol. 2015 Apr;5(4):276-82.

7 Hamilos DL. Chronic rhinosinusitis in patients with cystic fibrosis. J Allergy Clin Immunol Pract. 2016 Jul;4(4):605-12.

8 Noone PG, Leigh MW, Sannuti A, Minnix SL, Carson JL, Hazucha M, et al. Primary ciliary dyskinesia: diagnostic and phenotypic features. Am J Respir Crit Care Med. 2004 Feb; 169(4):459-67.

9 Lee RJ, Cohen NA. Bitter and sweet taste receptors in the respiratory epithelium in health and disease. J Mol Med (Berl). 2014 Dec; 92(12):1235-44.

10 Chandrashekar J, Mueller KL, Hoon MA, Adler E, Feng L, Guo W, et al. T2Rs function as bitter taste receptors. Cell. 2000 Mar; 100(6):703-11.

11 Zhang Y, Hoon MA, Chandrashekar J, Mueller KL, Cook B, Wu D, et al. Coding of sweet, bitter, and umami tastes: different receptor cells sharing similar signaling pathways. Cell. $2003 \mathrm{Feb} ; 112(3): 293-301$.

12 Chandrashekar J, Hoon MA, Ryba NJ, Zuker CS. The receptors and cells for mammalian taste. Nature. 2006 Nov;444(7117):288-94.

13 Tizzano M, Gulbransen BD, Vandenbeuch A, Clapp TR, Herman JP, Sibhatu HM, et al. Nasal chemosensory cells use bitter taste signaling to detect irritants and bacterial signals. Proc Natl Acad Sci USA. 2010 Feb;107(7): $3210-5$.

14 Pearson JP, Passador L, Iglewski BH, Greenberg EP. A second $\mathrm{N}$-acylhomoserine lactone signal produced by Pseudomonas aeruginosa. Proc Natl Acad Sci USA. 1995 Feb;92(5): $1490-4$.

15 Mfuna Endam L, Filali-Mouhim A, Boisvert P, Boulet LP, Bossé Y, Desrosiers M. Genetic variations in taste receptors are associated with chronic rhinosinusitis: a replication study. Int Forum Allergy Rhinol. 2014 Mar; 4(3):200-6.

16 Tournas A, Mfuna L, Bossé Y, Filali-Mouhim A, Grenier JP, Desrosiers M. A pooling-based genome-wide association study implicates the p73 gene in chronic rhinosinusitis. J Otolaryngol Head Neck Surg. 2010 Apr;39(2):18895.

17 Freund JR, Lee RJ. Taste receptors in the upper airway. World J Otorhinolaryngol Head Neck Surg. 2018 Mar;4(1):67-76.

18 Workman AD, Palmer JN, Adappa ND, Cohen NA. The Role of Bitter and Sweet Taste Receptors in Upper Airway Immunity. Curr Allergy Asthma Rep. 2015 Dec;15(12):72.

19 Douglas JE, Cohen NA. Taste Receptors Mediate Sinonasal Immunity and Respiratory Disease. Int J Mol Sci. 2017 Feb;18(2):E437.

20 Kimple AJ, Garland AL, Cohen SP, Setola V, Willard FS, Zielinski T, et al. RGS21, a regula- tor of taste and mucociliary clearance? Laryngoscope. 2014 Mar;124(3):E56-63.

21 Cohen SP, Buckley BK, Kosloff M, Garland AL, Bosch DE, Cheng G Jr, et al. Regulator of G-protein signaling-21 (RGS21) is an inhibitor of bitter gustatory signaling found in lingual and airway epithelia. J Biol Chem. 2012 Dec;287(50):41706-19.

22 Fokkens WJ, Lund VJ, Mullol J, et al. European Position Paper on Rhinosinusitis and Nasal Polyps 2012. Rhinol Suppl. 2012 Mar; 23:3 p preceding table of contents, 1-298.

23 Lund VJ, Mackay IS. Staging in rhinosinusitus. Rhinology. 1993 Dec;31(4):183-4.

24 DeConde AS, Mace JC, Bodner T, Hwang PH, Rudmik L, Soler ZM, et al. SNOT-22 quality of life domains differentially predict treatment modality selection in chronic rhinosinusitis. Int Forum Allergy Rhinol. 2014 Dec; 4(12):972-9.

251000 Genomes Project Consortium; Auton A, Brooks LD, et al. A global reference for human genetic variation. Nature 2015 Oct; 526(7571):68-74.

26 Gall BJ, Wilson A, Schroer AB, Gross JD, Stoilov P, Setola V, et al. Genetic variations in GPSM3 associated with protection from rheumatoid arthritis affect its transcript abundance. Genes Immun. 2016 Mar;17(2): 139-47.

27 Thorisson GA, Smith AV, Krishnan L, Stein LD. The international HapMap project web site. Genome Res. 2005 Nov;15(11):1592-3.

28 Salas A, Pardo-Seco J, Cebey-López M, Gómez-Carballa A, Obando-Pacheco P, RiveroCalle I, et al.; GENDRES network. Whole Exome Sequencing reveals new candidate genes in host genomic susceptibility to Respiratory Syncytial Virus Disease. Sci Rep. 2017 Nov;7(1):15888.

29 Reed DR, Zhu G, Breslin PA, Duke FF, Henders AK, Campbell MJ, et al. The perception of quinine taste intensity is associated with common genetic variants in a bitter receptor cluster on chromosome 12. Hum Mol Genet. 2010 Nov; 19(21):4278-85.

30 Kim UK, Jorgenson E, Coon H, Leppert M, Risch N, Drayna D. Positional cloning of the human quantitative trait locus underlying taste sensitivity to phenylthiocarbamide. Science. 2003 Feb;299(5610):1221-5.

31 Lee RJ, Cohen NA. Role of the bitter taste receptor T2R38 in upper respiratory infection and chronic rhinosinusitis. Curr Opin Allergy Clin Immunol. 2015 Feb;15(1):14-20.

32 Shah AS, Ben-Shahar Y, Moninger TO, Kline JN, Welsh MJ. Motile cilia of human airway epithelia are chemosensory. Science. 2009 Aug;325(5944):1131-4.

33 Chamoun E, Hutchinson JM, Krystia O, Mirotta JA, Mutch DM, Buchholz AC, et al.; Guelph Family Health Study. Single Nucleotide Polymorphisms in Taste Receptor Genes Are Associated with Snacking Patterns of Pre-
school-Aged Children in the Guelph Family Health Study: A Pilot Study. Nutrients. 2018 Jan;10(2):E153.

34 Pawellek I, Grote V, Rzehak P, Xhonneux A, Verduci E, Stolarczyk A, et al.; European Childhood Obesity Trial Study Group. Association of TAS2R38 variants with sweet food intake in children aged 1-6 years. Appetite. 2016 Dec;107:126-34.

35 Deshaware S, Singhal R. Genetic variation in bitter taste receptor gene TAS2R38, PROP taster status and their association with body mass index and food preferences in Indian population. Gene. 2017 Sep;627: 363-8.

36 Schroer AB, Gross JD, Kaski SW, Wix K, Siderovski DP, Vandenbeuch A, et al. Development of Full Sweet, Umami, and Bitter Taste Responsiveness Requires Regulator of G protein Signaling-21 (RGS21). Chem Senses. 2018 May;43(5):367-78.

37 Jaggupilli A, Singh N, Upadhyaya J, Sikarwar AS, Arakawa M, Dakshinamurti S, et al. Analysis of the expression of human bitter taste receptors in extraoral tissues. Mol Cell Biochem. 2017 Feb;426(1-2):137-47.

38 Siffert W, Forster P, Jöckel KH, Mvere DA, Brinkmann B, Naber C, et al. Worldwide ethnic distribution of the $G$ protein beta3 subunit $825 \mathrm{~T}$ allele and its association with obesity in Caucasian, Chinese, and Black African individuals. J Am Soc Nephrol. 1999 Sep;10(9): 1921-30.

39 Siffert W. G-protein beta3 subunit $825 \mathrm{~T}$ allele and hypertension. Curr Hypertens Rep. 2003 Feb;5(1):47-53.

$40 \mathrm{Hu}$ Q, Zhang SY, Liu F, Zhang XJ, Cui GC, Yu $\mathrm{EQ}$, et al. Influence of GNB3 C825T polymorphism on the efficacy of antidepressants in the treatment of major depressive disorder: A meta-analysis. J Affect Disord. 2015 Feb;172: 103-9.

41 Huang L, Shanker YG, Dubauskaite J, Zheng JZ, Yan W, Rosenzweig S, et al. Ggamma13 colocalizes with gustducin in taste receptor cells and mediates IP3 responses to bitter denatonium. Nat Neurosci. 1999 Dec;2(12): 1055-62.

42 Zha Z, Han X, Smith MD, Liu Y, Giguère PM, Kopanja D, et al. A Non-Canonical Function of $G \beta$ as a Subunit of E3 Ligase in Targeting GRK2 Ubiquitylation. Mol Cell. 2015 Jun; 58(5):794-803.

43 Zha Z, Han XR, Smith MD, Lei QY, Guan KL, Xiong Y. Hypertension-associated C825T polymorphism impairs the function of $\mathrm{G} \beta 3$ to target GRK2 ubiquitination. Cell Discov. 2016 Apr;2(1):16005.

44 Kaski SW, Brooks S, Wen S, Haut MW, Siderovski DP, Berry JH, et al. Four single nucleotide polymorphisms in genes involved in neuronal signaling are associated with Opioid Use Disorder in West Virginia. J Opioid Manag. 2019 Mar-Apr;15(2):103-9.
Variations in Chemosensory Pathway

Genes Associated with CRS
Int Arch Allergy Immunol 2019;180:72-77 DOI: $10.1159 / 000499875$ 